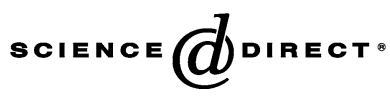

Journal of Controlled Release $\mathrm{xx}(2005) \mathrm{xxx}-\mathrm{xxx}$

\section{journal of controlled release}

www.elsevier.com/locate/jconrel

\title{
Transporting silence: Design of carriers for siRNA to angiogenic endothelium
}

\author{
Raymond M. Schiffelers ${ }^{\mathrm{a}, *}$, Archibald J. Mixson ${ }^{\mathrm{b}}$, Aslam M. Ansari ${ }^{\mathrm{c}}$, \\ Marcel H.A.M. Fens ${ }^{\mathrm{a}}$, Quingquan Tang ${ }^{\mathrm{c}}$, Qin Zhou ${ }^{\mathrm{c}}$, Jun $\mathrm{Xu}^{\mathrm{c}}$, Grietje Molema ${ }^{\mathrm{d}}$, \\ Patrick Y. Lu ${ }^{\mathrm{c}}$, Puthupparampil V. Scaria ${ }^{\mathrm{c}}$, Gert Storm ${ }^{\mathrm{a}}$, Martin C. Woodle \\ ${ }^{a}$ Department Pharmaceutics, Utrecht Institute for Pharmaceutical Sciences, Utrecht University, Utrecht, The Netherlands \\ ${ }^{\mathrm{b}}$ Department of Pathology, University of Maryland, Baltimore, MD, USA \\ ${ }^{\mathrm{c}}$ Intradigm Corporation, Rockville, MD, USA \\ ${ }^{\mathrm{d}}$ Department of Medical Biology, Pathology and Laboratory Medicine, Groningen University Institute for Drug Exploration, \\ Groningen University, Groningen, The Netherlands
}

Received 7 March 2005; accepted 19 May 2005

\begin{abstract}
The recently developed siRNA oligonucleotides are an attractive alternative to antisense as a therapeutic modality because of their robust, gene selective silencing of drug target protein expression. To achieve therapeutic success, however, several hurdles must be overcome including rapid clearance, nuclease degradation, and inefficient intracellular localization. In this presentation, we discuss design strategies for development of self-assembling nanoscale carriers for neovasculature targeted delivery of siRNA inhibiting tumor or ocular angiogenesis.
\end{abstract}

(C) 2005 Elsevier B.V. All rights reserved.

Keywords: RNA interference; siRNA; Drug targeting; Angiogenesis

\section{Introduction}

\subsection{Angiogenesis}

Angiogenesis is the formation of new blood vessels from pre-existing vasculature. It is an essential process

\footnotetext{
* Corresponding author. Tel.: +31 20 2539392; fax +31 30 2517839.

E-mail address: r.m.schiffelers@pharm.uu.nl (R.M. Schiffelers).
}

in normal physiology and tissue regeneration, but also one that plays a key role in several serious diseases. It is now well established as critical for tumor metastasis to distant tissues and for expansion of small clusters of malignant cells into a clinically relevant tumor [1-3]. Angiogenesis is also implicated in several inflammatory disorders including rheumatoid arthritis and psoriasis, and ocular diseases like diabetic retinopathy, age related macular degeneration and stromal keratitis [4-6]. The inflammatory process stimulates angiogenesis as part of the healing and regeneration of the

0168-3659/\$ - see front matter (C) 2005 Elsevier B.V. All rights reserved. doi:10.1016/j.jconrel.2005.05.018 
affected tissue and to recruit various cells of the immune system including lymphocytes and neutrophils $[7,8]$. Therapeutic interventions targeting angiogenesis are achieving clinical success and proving a valuable addition to the arsenal for management of a variety of malignant and inflammatory diseases $[9,10]$.

Although angiogenesis is a fairly complex and highly regulated process, the vascular endothelial growth factor (VEGF) biochemical pathway has been found to play an early key role. This growth factor is one of the strongest proteinaceous activators of angiogenesis, acting through binding of receptors VEGFR1 and VEGFR2 [11,12]. This pathway and the proteins involved constitute proven therapeutic targets for intervention to reduce angiogenesis. $[9,10,13]$.

\subsection{Targeting angiogenesis}

Activities of endothelial cells are at the root of the angiogenic process. Thus, drugs targeting angiogenesis biochemical pathways often must reach and act on these endothelial cells. Means to target drugs to these cells offer the prospect for focusing their activity at the key cell in angiogenesis-dependent diseases. The accessibility of these endothelial cells from the blood stream is an important advantage over efforts to target other cell types, such as tumor cells. Furthermore, angiogenic endothelial cells are phenotypically distinct from quiescent endothelium. A number of receptors have been identified that may be used to specifically deliver drugs to angiogenic endothelium [14]. Alpha v beta 3 integrins are among the most intensively investigated proteins that are overexpressed on angiogenic endothelial cells, partly because small molecular weight high affinity ligands have been identified for this integrin. It has been shown that disulfide-stabilized peptides containing an Arg-Gly-Asp (RGD)-motif can interact with high affinity and avidity to alpha v-integrins [15-17]. Other peptides that have been shown to specifically interact with angiogenic endothelial cells are peptides containing an NGR-motif (binding to aminopeptidase N [18]), ATWLPPR (binding to VEGFR2 [19-21]), and APRPG (binding an unknown target [22,23]).

\subsection{RNA interference}

The broad importance of angiogenesis in diseases, and growing clinical success of angiogenic interven- tions, makes it an attractive area for investigation of new therapeutic modalities [13]. RNAi is a recently developed technique to silence proteins in a sequencespecific manner by inhibiting mRNA and consequently reducing protein expression [24]. The functional mediator of RNA interference is a short dsRNA oligonucleotide called small interfering RNA (siRNA). A growing number of investigations are being reported on efforts to use siRNA as a candidate therapeutic agent. Most efforts reported to date are based on studies in rodents, and rodent models of disease. With the exception of local administration into eye tissues, the results show low activity due to a strong dependence on methods for intracellular delivery, as found for antisense and gene therapy approaches. One surprising observation is that siRNA composed of unmodified RNA is rapidly cleared by the kidneys [25], indicating low binding to proteins or cells in the blood and a surprising lack of nuclease degradation as the major barrier. A delivery strategy for use of siRNA as a therapeutic modality should therefore reduce glomerular filtration, firstly, and secondly optimize intracellular delivery to target cells, while also minimizing exposure to nuclease and distribution to non-target tissues. Nanoparticle delivery systems developed with a ligand-polymer-polymer conjugate appear attractive to meet these needs and indeed neovasculature targeted forms using a "cyclic" RGD peptide as the ligand have produced successful results [26,27]. In this report, the design elements for endothelium-targeted nanoparticles for delivery of siRNA are described.

\section{Materials and methods}

\subsection{RGD-PEG-PEI}

Synthesis of RGD-PEG-PEI was carried out in two steps as described [26]. Briefly, triethylamine (TEA) in tetrahydrofuran (THF) was added to a stirring solution of RGD-peptide in dimethylsulfoxide (DMSO) under a stream of nitrogen. After stirring for $1 \mathrm{~min}$, a solution of N-hydroxy succinimidyl-poly(ethylene glycol) (Mw 2500)-vinyl sulfone (Nektar Therapeutics, Huntsville, AL) in THF:DMSO was added. The reaction mixture was stirred at room temperature for 4 $\mathrm{h}$, and purified by HPLC. Conjugation was confirmed by Mass Spectral analysis (MALDI). In the second 
step, to a solution of RGD-peptide coupled PEGvinylsulfone in pure DMSO, TEA in THF was added. To the stirring reaction mixture, poly (ethylene imine) (PEI) (Mw $25 \mathrm{kD}$ ) was added and the reaction mixture was stirred overnight. The completion of the conjugation was checked by disappearance of RGDPEG-vinyl sulfone on thin layer chormatography. Degree of conjugation of RGD-PEG to PEI was determined by NMR spectrometry to be $7 \%$ of the PEIamines that were modified with RGD-PEG. The polymer was blended with unconjugated PEI at a molar ratio of $1: 1$ and mixed with siRNA in $10 \mathrm{mM}$ Hepes $\mathrm{pH} 7.4$ under vigorous vortexing to obtain polymer:siRNA complexes.

\subsection{RGD-PEG-DSPE:DOTAP:DOPE}

DOPE (dioleoyl-phosphatidylethanolamine) as neutral co-lipid and DOTAP (dioleoyl trimethylammonium propane) as cationic lipid component were from Avanti Polar Lipids (Alabaster, AL). Maleimide coupled poly (ethylene glycol) distearoyl phosphatidyl ethanolamine (Mal-PEG-DSPE) was obtained from Nektar Therapeutics (Huntsville, AL) and PEGDSPE was a generous gift from Lipoid GmbH (Ludwigshafen, Germany). Lipids were dissolved in chloroform:methanol $1: 1 \mathrm{v}: \mathrm{v}$, in a molar ratio of 1.43 : 1.43:0.07:0.07 respectively, and the solvent was evaporated under rotary evaporation. Lipids were hydrated in Hepes-buffered saline $\mathrm{pH}$ 7.4, followed by repeated freeze-thaw and extrusion through two stacked polycarbonate membranes with a final pore size of $0.05 \mu \mathrm{m}$. The liposome suspension was mixed with siRNA in $10 \mathrm{mM}$ Hepes $\mathrm{pH} 7.4$ under vigorous vortexing to obtain lipid: siRNA complexes.

\subsection{Branched HK-peptides}

The biopolymer core facility at the University of Maryland synthesized the histidine-lysine (HK) polymers on a Ranin Voyager synthesizer (PTI, Tucson, AZ) as previously described [28,29]. The branched polymers consist of HK-arms emanating from a lysine core in which the lysine core is uncharged. The polymers were then purified on an HPLC (Beckman, Fullerton, CA). HK-polymers were mixed with siRNA in $10 \mathrm{mM}$ Hepes $\mathrm{pH} 7.3$ under vigorous vortexing to obtain HK-polymer: siRNA complexes.

\subsection{Characterization of complexes}

Complexes were analyzed by dynamic light scattering to determine particle size on a Coulter N4plus particle size instrument (Beckman Coulter). $\xi$-potential measurements to determine surface charge were performed on a Coulter Delsa 440 SX. Latex beads with defined size and mobility were used as standards (Beckman Coulter, Miami, FL).

\subsection{FACS analysis of cell binding}

Nearly confluent human umbilical vein endothelial cells (HUVEC) were washed with phosphate-buffered saline (PBS) and detached using 1 mM EDTA in PBS. Cells were suspended in FACS-buffer that consisted of PBS supplemented with $1 \%$ bovine serum albumin, $1.26 \mathrm{mM} \mathrm{CaCl}_{2}, 0.81 \mathrm{mM} \mathrm{MgSO}_{4}$ of $4{ }^{\circ} \mathrm{C}$, centrifuged, counted, and $10^{5}$ cells were transferred to FACS tubes (BD Biosciences). Cells were incubated with $2 \mu \mathrm{g}$ fluorescently labeled siRNA complexed to the indicated carrier for $1 \mathrm{~h}$ at $4{ }^{\circ} \mathrm{C}$. After incubation, cells were washed, fixed in $4 \%$ buffered formaldehyde and analyzed on a FACS Calibur flow cytometer (BD Biosciences). Preincubation of cells with a 100-fold molar excess of unconjugated RGD-peptide for $30 \mathrm{~min}$ at $4{ }^{\circ} \mathrm{C}$ was used to determine the involvement of RGD in carrier binding. Cell binding was analyzed with WinMDI software version 2.8 (Joseph Trotter).

\subsection{In vivo injection and fluorescence microscopy}

Balb/c mice received a single iv injection of $40 \mu \mathrm{g}$ siRNA labeled with fluorescein isothiocyanate. At 15 min after injection, organs were excised and snap frozen in liquid nitrogen. $5 \mu \mathrm{m}$ section were cut on a cryostat and examined by fluorescence microscopy.

\section{Results and discussion}

\subsection{Evading non-target tissues}

The molecular weight of siRNA of approximately $15 \mathrm{kD}$ is below the size limit for glomerular filtration, similarly as described for antisense oligonucleotides (ODN) [25,30,31]. The antisense ODN have been 
shown to accumulate in the periglomerular space, possibly through the "oligonucleotide channel" of the brush border. In vivo pharmacokinetic studies with iv administration of siRNA have indeed shown that the kidneys are a major organ for uptake and excretion of siRNA within minutes after intravenous administration [25].

We have investigated the accumulation of fluorescently labeled siRNA after intravenous injection in kidneys of mice (Fig. 1A). Especially in the cortical region of the kidneys pronounced accumulation of fluorescence was visualized, which is similar to observations made for ODN [30]. The levels of fluorescence in this organ were highest of all tissues investigated, followed by levels in the liver (data not shown). Levels in all other tissues, including tumor tissue (Fig. 1C) were too low to be detected. These data suggest that uptake of siRNA by non-target tissues (most notably kidney and liver) is responsible for loss of the majority of the injected dose. Incubation in serum at $37{ }^{\circ} \mathrm{C}$ indicates stability of unmodified siRNA for many minutes $[25,26]$ and non-target tissue exposure can result in non-specific effects [32,33] although lacking interferon induction [34]. Therefore, evasion of nontarget tissue uptake is equally or more important than enhancement of siRNA stability.

Complexation of siRNA into nanoparticles should increase its apparent molecular weight, preventing glomerular filtration and/or oligonucleotide channel transport. Cationic complexing agents we have used for siRNA are shown in Fig. 2. Indeed, complexation of siRNA with the cationic lipid DOTAP reduced levels of fluorescence in the kidney (Fig. 1B), whereas levels in other tissues, like liver and spleen (data not shown) were increased. Also a remarkable increase in levels of fluorescence could be visualized in tumor tissue in the immediate vicinity of tumor capillaries (Fig. 1D).
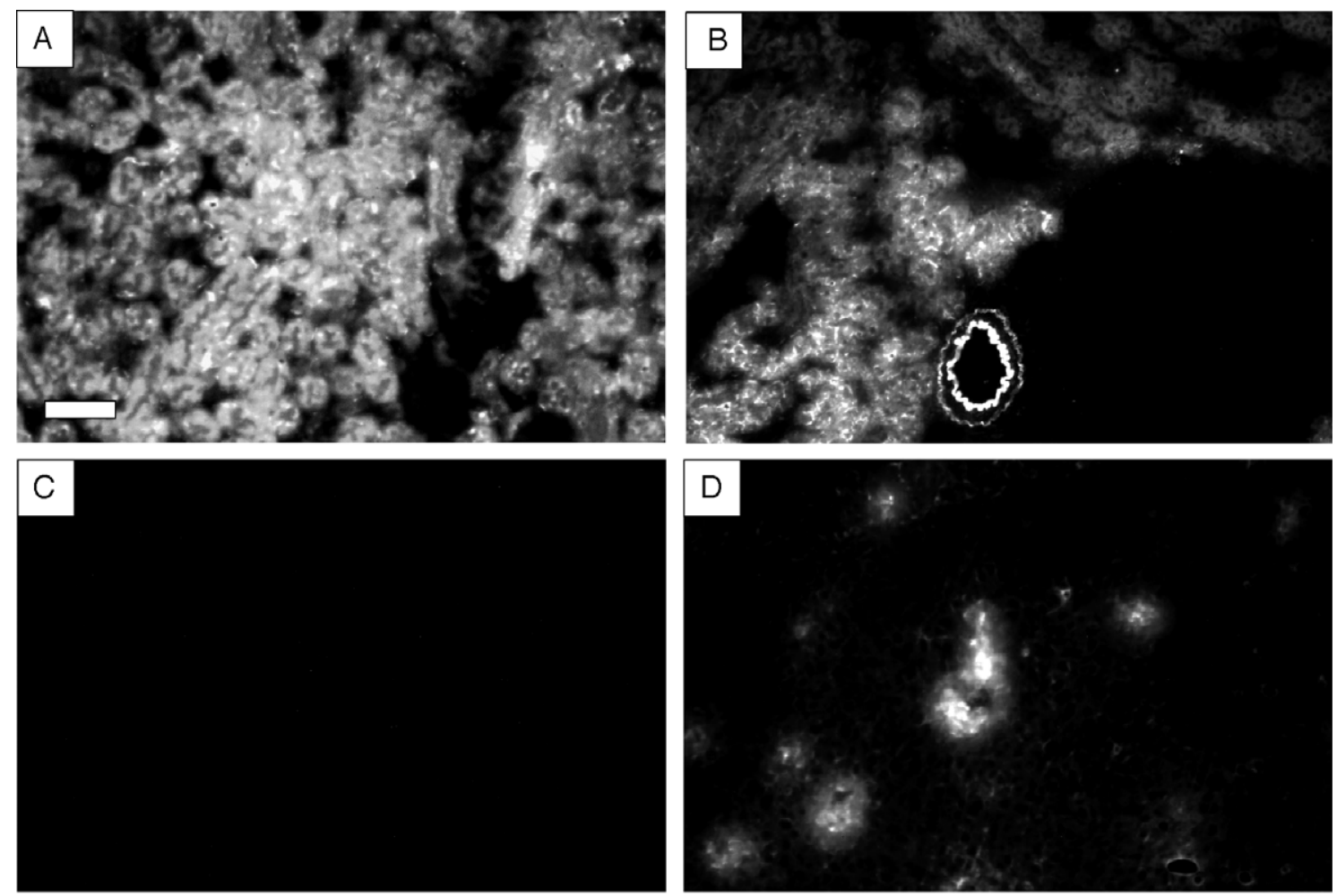

Fig. 1. FITC-siRNA accumulation in kidney and tumor tissue. FITC-siRNA was administered intravenously in the free form (A, C) or complexed to DOTAP (B, D). Fluorescence photomomicrographs are shown from kidney (A, B) and tumor tissue (C, D) dissected at 15 min post injection. Bar is $40 \mu \mathrm{m}$. 

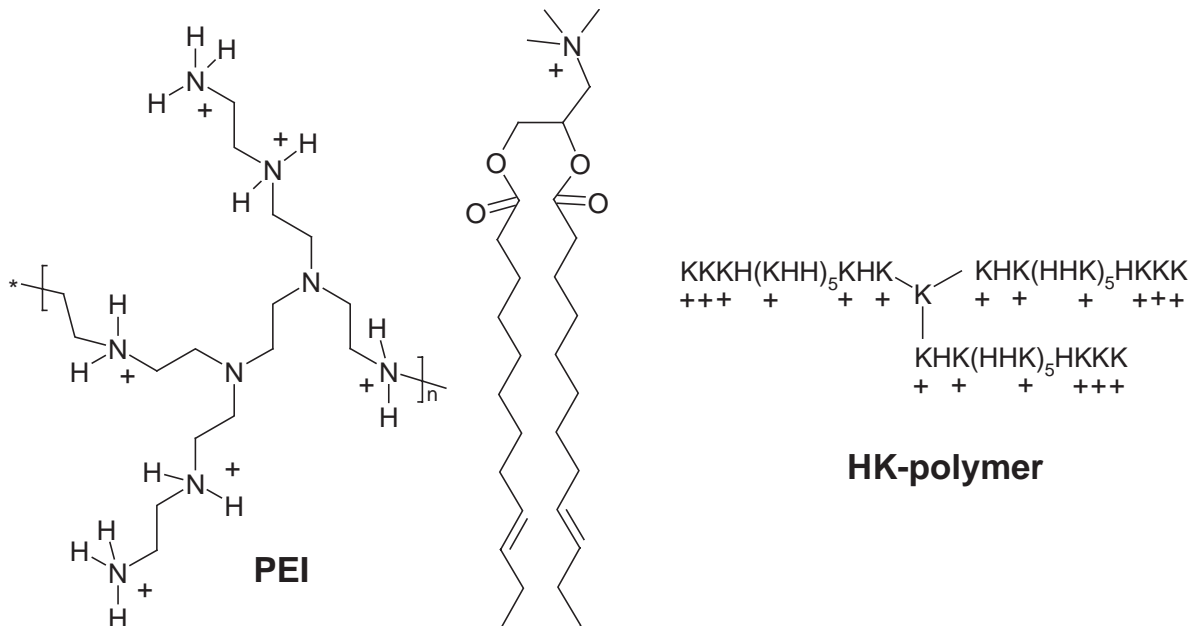

\section{HK-polymer}

Fig. 2. Cationic complexing agents for siRNA. Amphiphilic DOTAP is a phospholipid that bears a positive charge on the hydrophilic head group. HK-polymer is a branched histidine $(\mathrm{H})$-lysine $(\mathrm{K})$ polypeptide with positive charge on the lysine residues and somewhat on the histidine residues. The figure shows one possible HK polymer structure but the number of branches, and sequence patterns can be tailored for optimal transfection characteristics. Branched PEI (Mw $25 \mathrm{kD}$ ) bears positive charges on the primary and secondary amines.

These cationic agents can complex siRNA into nanoscale particles suitable for intravenous injection (Table 1).

PEI is one of the longest-standing polymers for non-viral nucleic acid delivery systems. Apart from a high charge density providing strong electrostatic interactions, PEI also has good properties for facilitating endosomal escape. One hypothesis for this enhancement of endosomal escape is that PEI acts as a 'proton sponge' during acidification of the endosome.

DOTAP has also been widely employed for nucleic acid delivery. It can be mixed with helper lipids (like dioleoyl phosphatidylethanolamine (DOPE)) that promote fusion of the particles with the endosomal membrane, aiding endosomal escape. Mixing with poly (ethylene glycol)-coupled phosphatidylethanolamine lipids can provide steric stabilization. Lipids may offer the advantage of biodegradability over PEI.

Biodegradability is also a feature of HK-polymers. Compared to PEI and liposomes, branched HK-poly- mers are relatively early in their development and most studies with siRNA have been done with unmodified HK-polymers in in vitro experiments. Nevertheless, modification of the HK polymers with PEG and RGD should be readily achievable (e.g., by addition of a cysteine to the terminal branches). The potential advantages of peptide-based therapy, which include HK-polymers, are that the amino acid building blocks, branching and sequence patterns can be easily manipulated to develop improved carriers for specific applications. For example, by changing the ratio and the sequence of histidine and lysine, transfection with HK polymers can favor siRNA delivery or plasmid delivery. With in vitro studies, highly branched $\mathrm{HK}$ polymers including $\mathrm{H}^{3} \mathrm{~K} 8 \mathrm{~b}$ (consisting of 8 branches with a repeating HHHK sequence) have been demonstrated to be very effective carriers of siRNA with low toxicity to cells [28]. Although branched HK polymers have not been used yet as carriers of siRNA for systemic in vivo studies, preliminary data demonstrate that unmodified HK poly- 
Table 1

Particle size and $\xi$-potential at the given $N / P$ ratio for PEI, cationic liposomes and HK-polymers complexed siRNA

\begin{tabular}{llll}
\hline Formulation & PEI & Liposomes & HK-polymer \\
\hline Size & $0.12 \pm 0.04 \mu \mathrm{m}$ & $0.21 \pm 0.05 \mu \mathrm{m}$ & $0.47 \pm 0.07 \mu \mathrm{m}$ \\
$\xi$-potential & $35 \pm 4 \mathrm{mV}$ & $41 \pm 9 \mathrm{mV}$ & $10 \pm 1 \mathrm{mV}$ \\
$N / P$ ratio & $2: 1$ & $4: 1$ & $4: 1$ \\
$n$ & 3 & 3 & 3 \\
Formulation & PEG-PEI & PEG-liposomes & \\
\hline Size & $0.09 \pm 0.01 \mu \mathrm{m}$ & $0.16 \pm 0.05 \mu \mathrm{m}$ & \\
$\xi$-potential & $5 \pm 6 \mathrm{mV}$ & $3 \pm 5 \mathrm{mV}$ & \\
$N / P$ ratio & $2: 1$ & $4: 1$ & \\
$n$ & 3 & 3 \\
Formulation & RGD-PEG-PEI & RGD-PEG-liposomes \\
\hline Size & $0.09 \pm 0.01 \mu \mathrm{m}$ & $0.14 \pm 0.03 \mu \mathrm{m}$ & \\
$\xi$-potential & $6 \pm 1 \mathrm{mV}$ & $7 \pm 4 \mathrm{mV}$ & \\
$N / P$ ratio & $2: 1$ & $4: 1$ & \\
$n$ & 3 & 5
\end{tabular}

Particle size was measured using dynamic light scattering. $\xi$-potential as a measure of surface charge was measured on a zeta-sizer, $N / P$ ratio is reported as ratio between number of positively charged amine groups $(N)$ of the cationic complexing agent and negatively charged phosphate groups $(P)$ of siRNA. $n=$ number of preparations.

mers are effective carriers of plasmids (which may include siRNA expressed by the H1 or U6 promoters) to a variety of tissues in vivo (unpublished data) and this suggests they will also be adaptable for siRNA oligonucleotides.

Cationic reagent-siRNA complexes can be made net positive, neutral, or negative depending on the ratio of the components in the complex. Net positive complexes (as shown in the table) are the most active in terms of siRNA silencing in cell culture [26-28]. A working hypothesis for this phenomenon is that electrostatic interactions between the negatively charged cell membrane and the positively charged particles increases cell uptake. This is a "non-specific" uptake since all cells have a similar negative membrane charge. It may also be that the mechanism is more related to binding positively charged serum/plasma components first before binding to the cells. Regardless of the underlying mechanism, the uptake takes place through interactions with a multitude of cell surface components since it is not specific but charge mediated. Thus a significant problem for systemic administration of these positively charged siRNAcomplexes is interaction with cells in many tissues.
One consequence is loss of a vast majority of the input dose to non-target tissues and cells. It is highly likely, if not certain, that some of these unwanted interactions will be toxic.

Our strategy is to minimize unwanted interactions by controlling the surface properties of the complexes. Reduction of non-specific binding is provided by a steric polymer coating on the particle. The hydrophilic polymer PEG is used in the PEI and lipid complexes (Fig. 3).

A steric coat can be introduced onto the complex surface by covalently conjugating the steric polymer onto the condensing agent before complexation with siRNA. The action of the steric coating is perhaps best appreciated when comparing the $\xi$-potential of unconjugated complexes with the conjugated complexes (i.e. PEI vs PEG-grafted PEI) (Table 1). The strong surface potential of unconjugated PEI-siRNA complexes of $35 \mathrm{mV}$ is reduced to a near neutral charge of $5 \mathrm{mV}$ for PEG-PEI:siRNA complexes with a concomitant loss of $>80 \%$ of binding to cell surfaces [26].

PEGylation of the cationic polymer before complexation seems a preferred method over conjugation of the polymer to a preformed complex. For one reason, chemical reactions carried out on the particle may damage the siRNA. For another reason, initial addition of steric polymers to the surface begins to form the barrier on the particle surface so that subsequent conjugation reactions are inhibited. This limits the amount of polymer that can be conjugated to the complex surface.
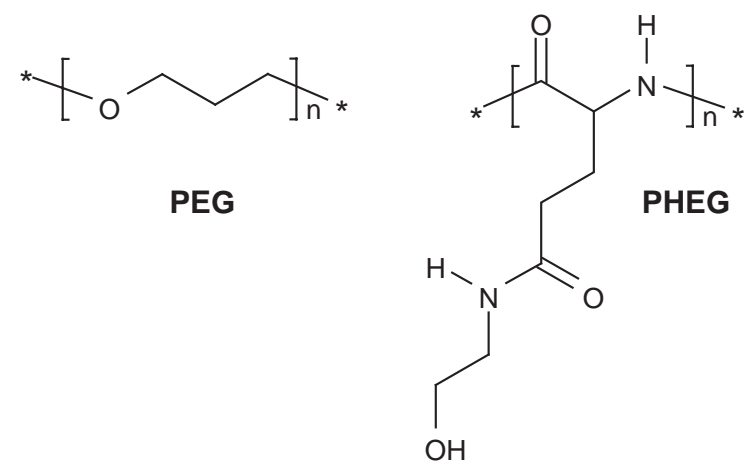

Fig. 3. Structure of poly(ethylene glycol) (PEG) and poly(hydroxy ethyl-L-glutamine (PHEG). 


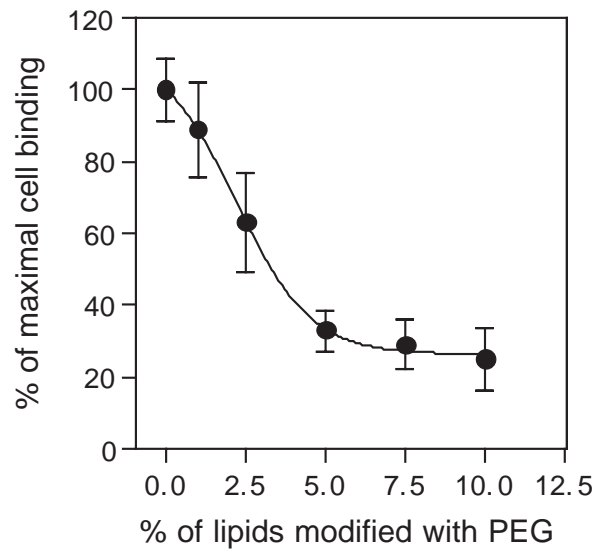

Fig. 4. Percentage of maximal cell binding of PEG-coated cationic lipid:siRNA complexes as a function of PEG-density. Liposomes were incubated with COS-7 cells at a concentration of $1 \mu \mathrm{mol}$ lipid/ $\mathrm{ml}$. Liposomes were prepared with equal amounts of DOTAP: DOPE and an indicated percentage of PEG-DSPE. $N / P$ ratio was $4: 1$ for all preparations. Binding was quantified using FITC-labeled siRNA. $n=3$, mean $+/-$ standard deviation.

For PEG-PEI, PEG is conjugated at random to multiple sites on the PEI using a stable covalent linkage. The grafting density can be varied. For lipid based carrier systems the PEG is conjugated to a phospholipid that will insert in the liposomal membrane that is subsequently complexed to siRNA. For both cationic liposome complexes and PEI-complexes approximately $5-10 \%$ modification of amines (for PEI) or phospholipids (for liposomes) with PEG appears to provide adequate shielding of the complex surface [26] and Fig. 4.

Alternative steric polymers, like poly hydroxy ethyl-L-glutamine (PHEG), have been prepared and are currently tested (Fig. 3). It is believed that these polymers should adopt a random configuration in solution and have specific molecular properties, such as low interfacial energy, hydrophilicity and high flexibility, to act as a steric stabilizer.

The loss in non-specific interactions makes the particles relatively inert. Successful formation of a steric polymer layer in terms of biological function can be observed from blood pharmacokinetics of the complexes following intravenous administration. The prolonged presence of complexes in the blood circulation can be exploited to increase targeting to pathological sites with an enhanced permeability, like malignant and inflamed tissue. Nevertheless, the for- mation of the steric polymer layer will have rendered the complex inactive in both cell culture and following intravenous administration [26].

\subsection{Interaction with target cell type}

After prolonged circulation the next step is restoring activity at the desired tissues and cells. In the absence of a steric coat, cationic surface charge helps to bind the target cell. This binding is lost when a steric polymer coat is formed and cellular binding must be replaced by another mechanism. In our prototypes, activity toward target cells has been restored using ligand-mediated binding and uptake. Careful selection of the target cell type and receptor is important in view of the accessibility of the cell type and efficiency of siRNA transfection. Angiogenic endothelial cells have the advantage of high accessibility from the blood stream and an ability to influence the disease process after transfection of a relatively small number of cells. In addition, alpha-v integrins are strongly overexpressed on these cells and are internalized, thereby allowing the siRNA to reach the cell interior. For this purpose "cyclic" RGD-peptides have been conjugated to the distal end of the steric polymer to mediate binding of the complexes with the integrins (Table 1, Fig. 5). The figure shows that both carrier systems can be targeted using the RGD-peptides to HUVEC and

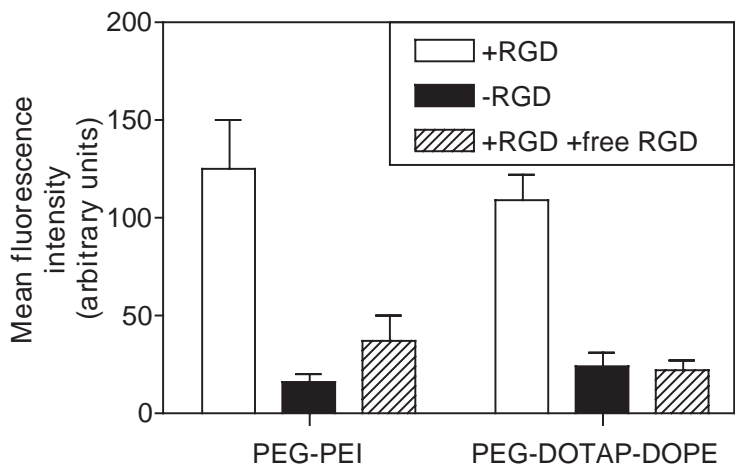

Fig. 5. Binding of siRNA carriers to HUVEC. $10^{5}$ HUVEC were incubated with $2 \mu \mathrm{g}$ FITC-labeled siRNA formulated with PEG-PEI or PEG-liposomes, with or without targeting RGD-peptide in the presence or absence of an excess of free RGD-peptide. Complexes were incubated for $1 \mathrm{~h}$ at $4{ }^{\circ} \mathrm{C}$. After the incubation period, the cells were washed, fixed with $4 \%$ buffered formaldehyde and cell-bound fluorescence was analyzed by FACS analysis. 


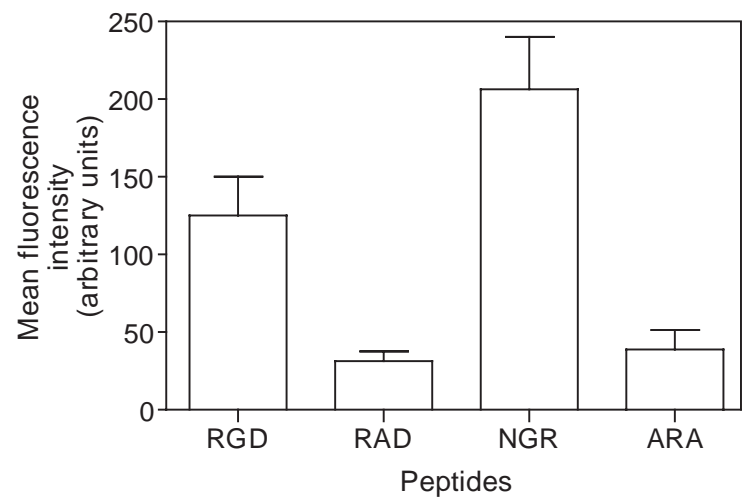

Fig. 6. Binding of RGD or NGR modified PEG-liposomes complexed to siRNA. $10^{5}$ HUVEC were incubated with $2 \mu \mathrm{g}$ FITClabeled siRNA formulated with RGD-PEG-liposomes or NGRPEG-liposomes (RAD and ARA-peptide functioned as non-binding controls). Complexes were incubated for $1 \mathrm{~h}$ at $4{ }^{\circ} \mathrm{C}$. After the incubation period, the cells were washed, fixed with $4 \%$ buffered formaldehyde and cell-bound fluorescence was analyzed by FACS analysis.

that the interaction with cells is dependent on the presence of the targeting ligand.

The targeting ligand can be chosen to address other receptors on angiogenic endothelial cells. This is important in view of the pronounced heterogeneity in gene expression of endothelial cells in vivo. This profile constantly changes. Sequentially, endothelial cells become activated, dissolve the basal membrane, proliferate, migrate and finally mature into functional blood vessels by remodeling, tube formation and vascular stabilization. Each phase is associated with expression of specific proteins and can (theoretically) be targeted with the appropriate targeting ligand or a combination of ligands. We have demonstrated the flexibility of the system by attaching alternative targeting ligands. Fig. 6 shows an example of PEGliposomes modified with NGR peptide that has a high affinity for aminopeptidase $\mathrm{N}$ overexpressed on angiogenic endothelium.

The RGD-PEG-PEI carriers have been tested in different animal models of pathological angiogenesis $[26,27]$. siRNA delivered to angiogenic endothelium was able to silence expression of reporter genes in vitro, and silence expression of transfected VEGF, VEGFR1 or VEGFR2 in a sequence-specific manner. In N2A tumor-bearing mice intravenous administration with siRNA targeting VEGFR2 showed tumor selective activity including inhibition of gene expression, tumor angiogenesis, and tumor growth. The inhibition of tumor growth was reflected by a marked loss of peritumoral vascularization [26].

In mice suffering pathological neovascularization in the eye resembling herpetic stromal keratitis, siRNAs targeting either VEGF, VEGFR1, VEGFR2 or a mix of the three were used. Local or systemic administration of siRNAs significantly inhibited neovascularization. The efficacy of systemic administration was superior when using RGD-PEG-PEI to deliver siRNA. Mixtures of siRNAs targeting three genes in the VEGF pathway surpassed the effects of each gene targeted siRNAs alone when administered locally or systemically in RGD-PEG-PEI against ocular angiogenesis [27].

The strong activity of neovascular tissue-targeted siRNA nanoparticles in two models of pathological angiogenesis offers promise for the use of siRNA and ligand-directed nanoparticle formulations as new therapeutic agents to control unwanted angiogenesis. The flexibility of siRNA to target various factors in the angiogenic process by simply changing siRNA sequence has important advantages for therapeutic application. In addition, the modular character of the carrier system elements, such as ligand, steric polymer and carrier, allows tailoring of the nanoparticle carrier characteristics to achieve optimal target cell selectivity. To date, more than six splice variants of VEGF have been identified which seem to have different functions in promoting angiogenesis $[35,36]$. In this perspective, site-specific delivery of siRNA to inhibit VEGF signaling may offer a way to silence multiple proteins in this pathway and perhaps even to silence the specific splice variants responsible for the pathology.

\section{Acknowledgments}

Parts of this study was funded by the Dutch Cancer Society grant 2000-2185 and by the National Institutes of Health (USA).

\section{References}

[1] P. Carmeliet, Angiogenesis in health and disease, Nat. Med. 9 (2003) 653-660.

[2] Y. Cao, Antiangiogenic cancer therapy, Semin. Cancer Biol. 14 (2004) 139-145. 
[3] M. Kirsch, G. Schackert, P.M. Black, Metastasis and angiogenesis, Cancer Treat. Res. 117 (2004) 285-304.

[4] H.G. Augustin, Translating angiogenesis research into the clinic: the challenges ahead, Br. J. Radiol. 76 (1) (2003) S3-S10 (Spec. No.).

[5] M. Ziche, S. Donnini, L. Morbidelli, Development of new drugs in angiogenesis, Curr. Drug Targets 5 (2004) 485-493.

[6] A.W. Griffioen, G. Molema, Angiogenesis: potentials for pharmacologic intervention in the treatment of cancer, cardiovascular diseases, and chronic inflammation, Pharmacol. Rev. 52 (2000) 237-268.

[7] X.W. Bian, J.H. Chen, X.F. Jiang, J.S. Bai, Q.L. Wang, $X$. Zhang, Angiogenesis as an immunopharmacologic target in inflammation and cancer, Int. Immunopharmacol. 4 (2004) $1537-1547$.

[8] R.O. Schlingemann, Role of growth factors and the wound healing response in age-related macular degeneration, Graefes Arch. Clin. Exp. Ophthalmol. 242 (2004) 91-101.

[9] J. Baselga, L. Gianni, C. Geyer, E.A. Perez, A. Riva, C. Jackisch, Future options with trastuzumab for primary systemic and adjuvant therapy, Semin. Oncol. 31 (5 Suppl 10) (2004) $51-57$

[10] Eyetech Study Group, Anti-vascular endothelial growth factor therapy for subfoveal choroidal neovascularization secondary to age-related macular degeneration: phase II study results, Ophthalmology 110 (2003) 979-986.

[11] D. Ribatti, The crucial role of vascular permeability factor/ vascular endothelial growth factor in angiogenesis: a historical review, Br. J. Haematol. 128 (2005) 303-309.

[12] T. Tammela, B. Enholm, K. Alitalo, K. Paavonen, The biology of vascular endothelial growth factors, Cardiovasc. Res. 65 (2005) 550-563.

[13] P.Y. Lu, F.Y. Xie, M.C. Woodle, Modulation of angiogenesis with siRNA inhibitors for novel therapeutics, Trends Mol. Med. 11 (3) (2005 Mar) 104-113.

[14] R.M. Schiffelers, M.H. Fens, A.P. Janssen, G. Molema, G. Storm, Liposomal targeting of angiogenic vasculature, Curr. Drug Del. (in press).

[15] R.J. Kok, A.J. Schraa, E.J. Bos, H.E. Moorlag, S.A. Asgeirsdottir, M. Everts, D.K. Meijer, G. Molema, Preparation and functional evaluation of RGD-modified proteins as alpha(v)beta(3) integrin directed therapeutics, Bioconjug. Chem. 13 (2002) 128-135.

[16] A.P. Janssen, R.M. Schiffelers, T.L. ten Hagen, G.A. Koning, A.J. Schraa, R.J. Kok, G. Storm, G. Molema, Peptide-targeted PEG-liposomes in anti-angiogenic therapy, Int. J. Pharm. 254 (2003) $55-58$.

[17] A.J. Schraa, R.J. Kok, S.M. Botter, S. Withoff, D.K. Meijer, L.F. de Leij, G. Molema, RGD-modified anti-CD3 antibodies redirect cytolytic capacity of cytotoxic T-lymphocytes toward alphavbeta3-expressing endothelial cells, Int. J. Cancer 112 (2004) 279-285.

[18] F. Pastorino, C. Brignole, D. Marimpietri, M. Cilli, C. Gambini, D. Ribatti, R. Longhi, T.M. Allen, A. Corti, M. Ponzoni, Vascular damage and anti-angiogenic effects of tumor vesseltargeted liposomal chemotherapy, Cancer Res. 63 (2003) $7400-7409$.
[19] F. Ye, H.Z. Chen, X. Xie, D.F. Ye, W.G. Lu, Z.M. Ding, Vascular endothelial growth factor (VEGF) and ovarian carcinoma cell supernatant activate signal transducers and activators of transcription (STATs) via VEGF receptor-2 (KDR) in human hemopoietic progenitor cells, Gynecol. Oncol. 94 (2004) 125-133.

[20] R.Z. Renno, Y. Terada, M.J. Haddadin, N.A. Michaud, E.S. Gragoudas, J.W. Miller, Selective photodynamic therapy by targeted verteporfin delivery to experimental choroidal neovascularization mediated by a homing peptide to vascular endothelial growth factor receptor-2, Arch. Ophthalmol. 122 (2004) 1002-1011.

[21] R. Binetruy-Tournaire, C. Demangel, B. Malavaud, R. Vassy, S. Rouyre, M. Kraemer, J. Plouet, C. Derbin, G. Perret, J.C. Mazie, Identification of a peptide blocking vascular endothelial growth factor (VEGF)-mediated angiogenesis, EMBO J. 19 (2000) 1525-1533.

[22] N. Maeda, Y. Takeuchi, M. Takada, Y. Sadzuka, Y. Namba, N. Oku, Anti-neovascular therapy by use of tumor neovasculature-targeted long-circulating liposome, J. Control. Release 100 (2004) 41-52.

[23] N. Maeda, Y. Takeuchi, M. Takada, Y. Namba, N. Oku, Synthesis of angiogenesis-targeted peptide and hydrophobized polyethylene glycol conjugate, Bioorg. Med. Chem. Lett. 14 (2004) 1015-1017.

[24] K. Huppi, S.E. Martin, N.J. Caplen, Defining and assaying RNAi in mammalian cells, Mol. Cell 17 (2005) 1-10.

[25] D.A. Braasch, Z. Paroo, A. Constantinescu, G. Ren, O.K. Oz, R.P. Mason, D.R. Corey, Biodistribution of phosphodiester and phosphorothioate siRNA, Bioorg. Med. Chem. Lett. 14 (2004) 1139-1143

[26] R.M. Schiffelers, A. Ansari, J. Xu, Q. Zhou, Q. Tang, G. Storm, G. Molema, P.Y. Lu, P.V. Scaria, M.C. Woodle, Cancer siRNA therapy by tumor selective delivery with ligand-targeted sterically stabilized nanoparticle, Nucleic Acids Res. 32 (2004) e149.

[27] B. Kim, Q. Tang, P.S. Biswas, J. Xu, R.M. Schiffelers, F.Y. Xie, A.M. Ansari, P.V. Scaria, M.C. Woodle, P.Y. Lu, B.T. Rouse, Inhibition of ocular angiogenesis by siRNA targeting vascular endothelial growth factor pathway genes: therapeutic strategy for herpetic stromal keratitis, Am. J. Pathol. 165 (2004) 2177-2185.

[28] Q.X. Leng, P. Scaria, J. Zhu, A.J. Mixson, Highly branched HK peptides are effective carriers of siRNA, J. Gene Med. (in press).

[29] L. Zhang, N. Ambulos, A.J. Mixson, DNA delivery to cells in culture using peptides, Methods Mol. Biol. 245 (2004) $33-52$.

[30] B. Hanss, E. Leal-Pinto, L.A. Bruggeman, T.D. Copeland, P.E. Klotman, Identification and characterization of a cell membrane nucleic acid channel, Proc. Natl. Acad. Sci. U. S. A. 95 (1998) 1921-1926.

[31] B. Kühnast, F. Dollé, S. Terrazzino, B. Rousseau, C. Loch, F. Vaufrey, F. Hinnen, I. Doignon, F. Pillon, C. David, C. Crouzel, B. Tavitian, General method to label antisense oligonucleotides with radioactive halogens for pharmacological and imaging studies, Bioconjug. Chem. 11 (2000) 627-636. 
[32] S. Filleur, A. Courtin, S. Ait-Si-Ali, J. Guglielmi, C. Merle, A. Harel-Bellan, P. Clezardin, F. Cabon, siRNA-mediated inhibition of vascular endothelial growth factor severely limits tumor resistance to antiangiogenic thrombospondin-1, Can. Res. 63 (2003) 3919-3922.

[34] J.D. Heidel, S. Hu, X.F. Liu, T.J. Triche, M.E. Davis, Lack of interferon response in animals to naked siRNAs, Nat. Biotechnol. 22 (2004) 1579-1582.

[33] S. Agrawal, E.R. Kandimalla, Antisense and siRNA as agonists of toll-like receptors, Nat. Biotech. 22 (2004) 1533-1537.

[35] J. Woolard, W.Y. Wang, H.S. Bevan, Y. Qiu, L. Morbidelli, R.O. Pritchard-Jones, T.G. Cui, M. Sugiono, E. Waine, R.
Perrin, R. Foster, J. Digby-Bell, J.D. Shields, C.E. Whittles, R.E. Mushens, D.A. Gillatt, M. Ziche, S.J. Harper, D.O. Bates, VEGF165b, an inhibitory vascular endothelial growth factor splice variant: mechanism of action, in vivo effect on angiogenesis and endogenous protein expression, Cancer Res. 64 (2004) $7822-7835$.

[36] J. Grunstein, J.J. Masbad, R. Hickey, F. Giordano, R.S. Johnson, Isoforms of vascular endothelial growth factor act in a coordinate fashion to recruit and expand tumor vasculature, Mol. Cell. Biol. 20 (2000) 7282-7291. 